\title{
STARS4ALL Night Sky Brightness Photometer
}

\author{
Jaime Zamorano 1,*, Cristóbal García², Rafael González², Carlos Tapia , Alejandro \\ Sánchez de Miguel $^{1}$, Sergio Pascual ${ }^{1}$, Jesús Gallego ${ }^{1}$, \\ Esteban González ${ }^{3}$, Pablo Picazo ${ }^{1}$, Jaime Izquierdo ${ }^{1}$, Mireia Nievas ${ }^{1}$, Lucía García ${ }^{1}$, \\ Oscar Corcho $^{3}$, and The STARS4ALL consortium \\ ${ }^{1}$ Universidad Complutense de Madrid, 28040 Madrid, Spain \\ ${ }^{2}$ Agrupación Astro Henares, 28820 Coslada, Madrid, Spain \\ ${ }^{3}$ Universidad Politécnica de Madrid, 28660 Boadilla del Monte, Madrid, Spain
}

Received 21 November 2016, Accepted 12 December 2016

\begin{abstract}
We present the main features of TESS-W, the first version of a series of inexpensive but reliable photometers that will be used to measure night sky brightness. The bandpass is extended to the red with respect of that of the Sky Quality Meter (SQM). TESS-W connects to a router via WIFI and it sends automatically the brightness values to a data repository using Internet of Things protocols. The device includes an infrared sensor to estimate the cloud coverage. It is designed for fixed stations to monitor the evolution of the sky brightness. The photometer could also be used in local mode connected to a computer or tablet to gather data from a moving vehicle. The photometer is being developed within STARS4ALL project, a collective awareness platform for promoting dark skies in Europe, funded by the EU. We intend to extend the existing professional networks to a citizen-based network of photometers.
\end{abstract}

Keywords: Light pollution, Photometry, Sky brightness, Instrumentation

\section{Introduction}

Light pollution (the introduction by humans, directly or indirectly, of artificial light into the environment) is a major issue worldwide. One of the effects of the light pollution is the brightening of the night sky. The increase of the nocturnal sky glow prevents citizens from observing a dark starry sky. Although the light sources are located mainly in urban areas it is necessary to travel far from these places to find a sky with unpolluted, natural sky [1], [2].

The professional astronomical observatories are being pushed to remote areas as far as possible from the light pollution sources of the urban areas and efforts are made to prevent the increase of light pollution in the observatory surroundings. Moreover, the night sky brightness is one of the key parameters of the sky astronomical quality. The measure of the sky brightness as a proxy of light pollution has been the task of the astronomers to monitor the increasing light pollution that threatens their astronomical observatories.

The methods of the astronomical photometry, using the stars as standard sources, are lengthy and complicated. During the last decade there have been an increasing number of astronomers and interested citizens who have been measuring the night sky brightness using portable photometers. Their measurements were performed both under dark and bright skies. The Sky Quality Meter (SQM) from Unihedron is by far the most used [3]. Thousands of measures made with handheld SQM or with computer linked photometers have provided the data needed to build the world atlas of the sky brightness [4].

STARS4ALL, a collective awareness platform for promoting dark skies in Europe, funded by the EU [5], aims to push citizen science in light pollution. We are trying to involve citizens within the several light pollution

\footnotetext{
* Zamorano J, Tel.: +34-91-394-4590

E-mail address: jzamorano@fis.ucm.es
} 
initiatives (LPIs). One of them is related to development of a European Photometer Network. Interested citizens will help us in monitoring the light pollution of European villages with a sensor network. The network will extend the current existing professional photometer networks to a citizen-based network of photometers.

\section{Night Sky brightness photometer}

The TESS-W photometer is the first version of a series of cheap but reliable photometers that will be used to measure night sky brightness. TESS stands for 'telescope encoder and sky sensor' and has been developed by the amateur astronomer and expert engineer Cristóbal García as part of its remote observatory system (http://www.observatorioremoto.com/). The TESS-W photometer uses WiFi communication.

This version has been designed to monitor the sky from fixed places, but could also be used from a moving vehicle. This device also measures the ambient and sky temperature to record the cloud coverage. It communicates to a router via a WIFI module and sends automatically the brightness values to our data repository using IoT (Internet of Things) protocols. TESS-W does not need an additional enclosure since the box (80x55x35 mm) is weatherproof. The hardware, the software and the data will be open.

\subsection{Hardware}

The sky brightness detector is a TSL237 photodiode that convers light to frequency. It is the same sensor used by the SQM photometers. However, the bandpass is more extended to the red range with the use a dichroic filter. The light from the sky is collected with the optics that includes a dichroic filter to select the bandpass (see Figure 1). The filter fully covers the collector (1); the sensor (not seen in Figure 1) is located on a printed circuit board along with the custom-made electronics (2); the WiFi module (3) with an antenna inside the box that extends the WiFi range; a near-infrared sensor (4) is used to measure sky temperature; finally, the heater (5) is switched on when needed to get rid of condensation on the window (6). The field of view (FoV) is FWHM=17 degrees.
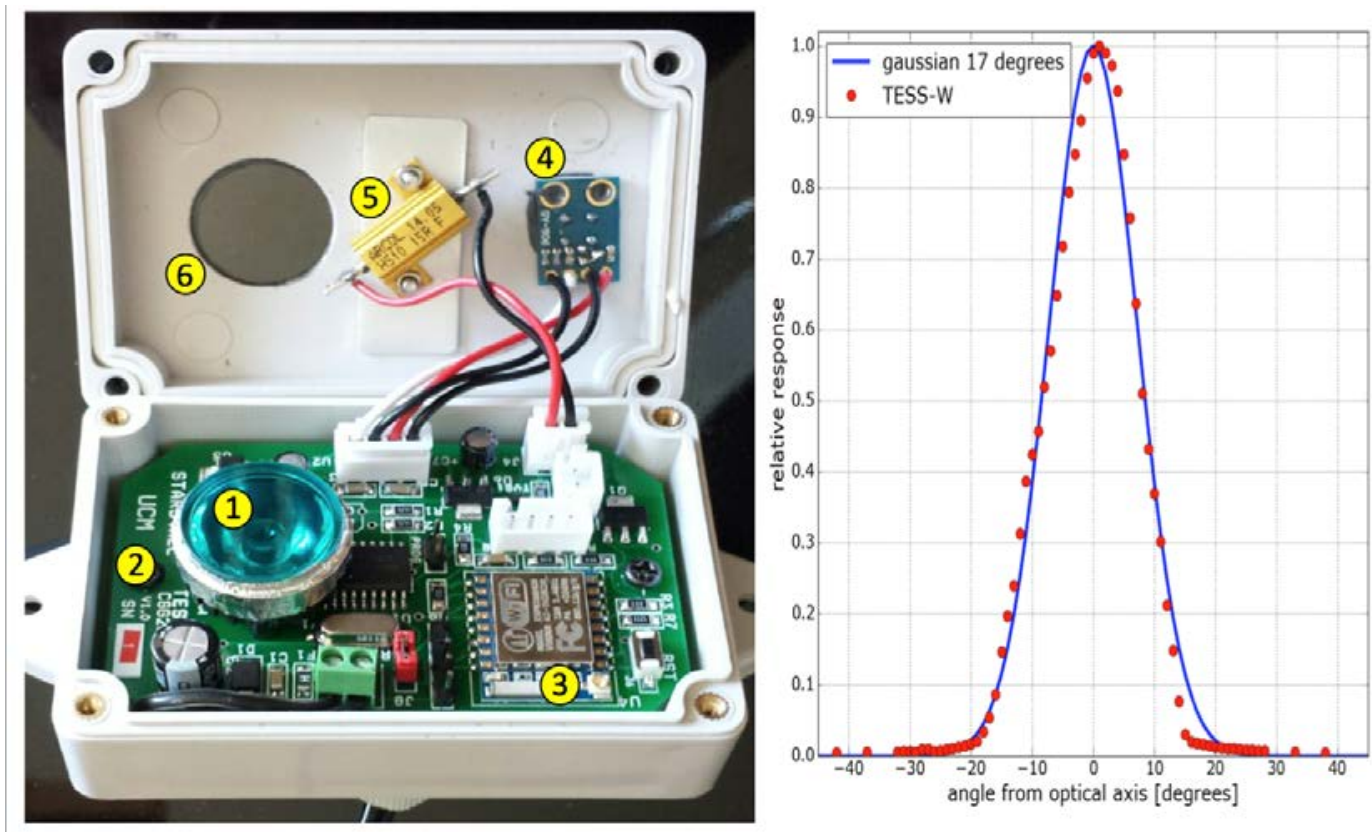

Fig. 1. (Left) The inside of enclosure with the optics and electronics of the TESS-V1 photometer. See text for description. (Right) TESS-W photometer field of view (FoV) is similar to a gaussian function of FWHM=17 degrees.

\subsection{Software}

The photometer is configured using a web browser to connect via WiFi to a router (SSID, password, name, interval between measures etc.). In normal use the reading of the sensors are received by the MQTT broker and stored. This is the method for monitor the sky brightness at fixed stations. The user can also read the data of its device in local mode with a computer or Android device. This method is useful for measures from a moving 
vehicle to map the sky brightness of extended regions. The GPS data is provided by the Android device that is connected to the photometer.
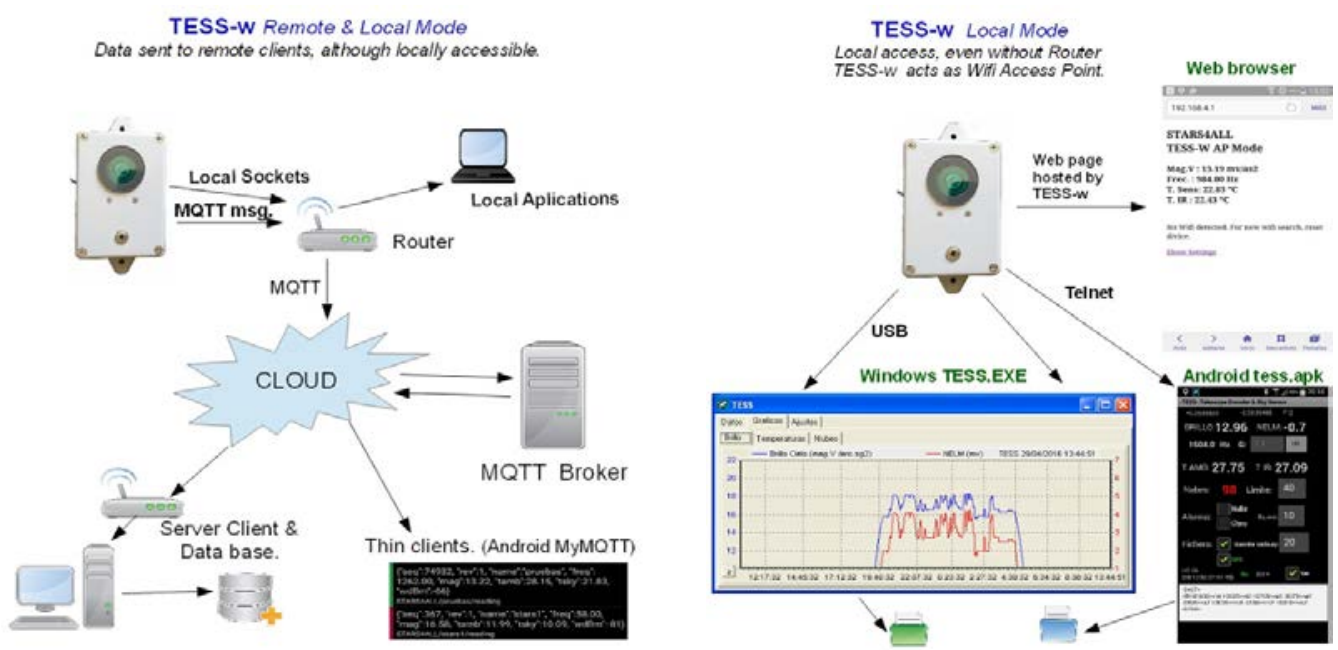

Fig. 2. (Left) Communication schematic. Local mode (Right)

\subsection{Spectral response}

Although the sensor employed is the same that uses SQM, we use a dichroic filter instead the color filter. The overall response is thus different and fully includes the lines of the High Pressure Sodium (HPS) lamps since the spectral response is extended to the red. We show in Figure 3 a comparison of the spectral responses of SQM and TESS-W photometers. Typical night sky spectrum from Calar Alto observatory [6] and Madrid (UCM observatory) [7] are shown for reference.
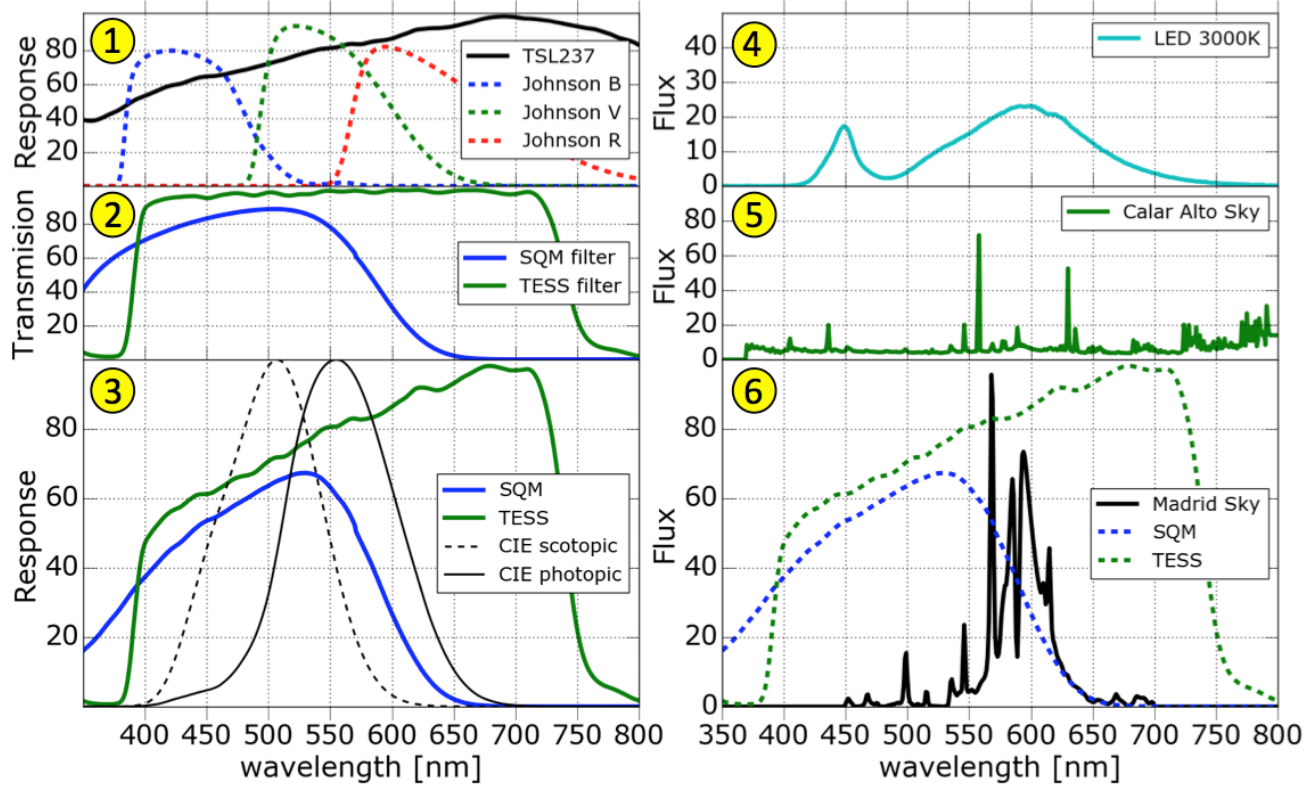

Fig. 3. (1) Spectral response of the TSL237 sensor. (2) Transmission of the HOYA CM-500 (BG39) used by Unihedron

SQM and that of the dichroic filter used in TESS. (3) Combined response of filter and sensor. (4) 3000K LED spectrum.

(5) Calar Alto Sky spectrum [6] and (6) Spectral response over spectrum of Madrid (SAND spectrometer [7]) 
The IR sensor allow us to estimate the cloud cover at the same time as the sky brightness is being measured. This is very important to discard measures of sky brightness affected by the light reflected into the clouds and to get a better statistical estimate of the sky brightness evolution [1],[2].

\section{Field tests}

The first 12 working units of the TESS photometer have been assembled and they are being tested under both bright and dark skies. Most of the locations are fixed stations of the Spanish Network of Light Pollution Studies which provides SQM readings for inter comparison. The absolute calibration will be performed on LICA-UCM optical lab and the astronomical observatory of the Universidad Complutense de Madrid.
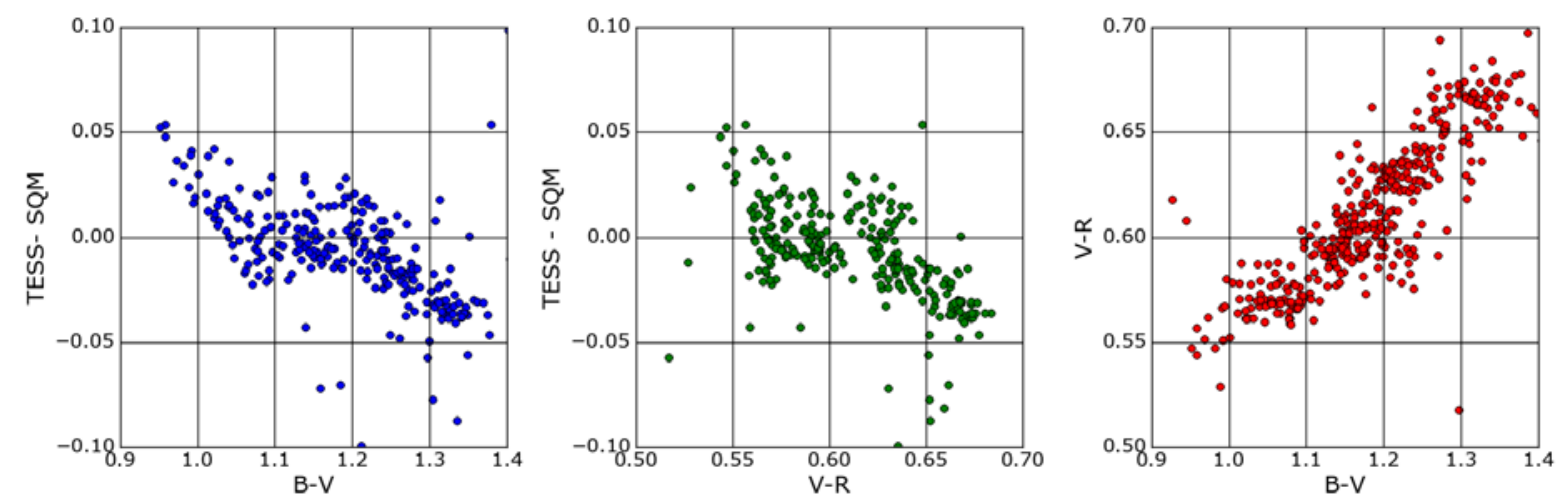

Fig. 4. NSB data obtained at UCM observatory using AstMon astronomical camera, SQM and TESS along one night.

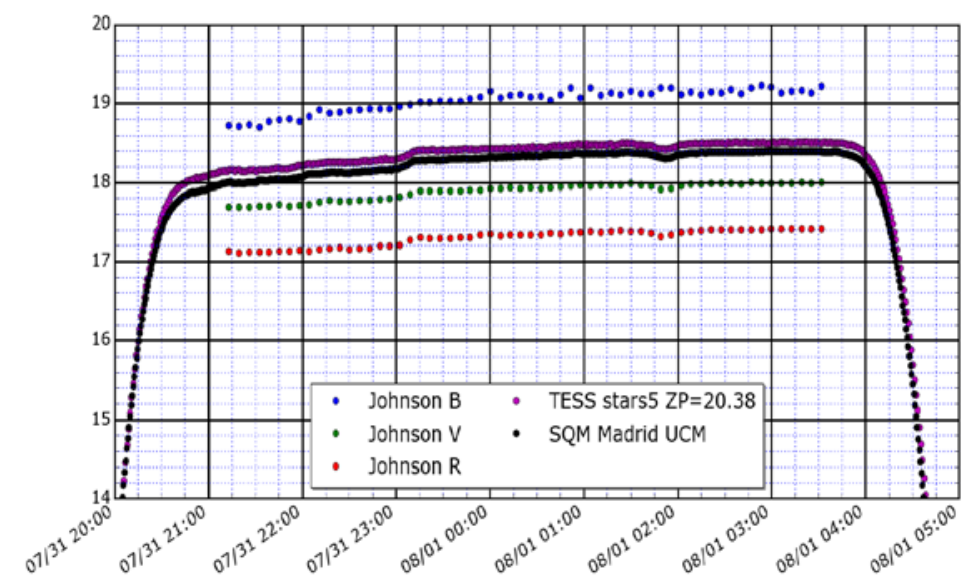

Fig. 5. Dependence of differences between TESS and SQM with the sky color.

We show in Figure 4 the measures of night sky brightness at zenith taken from UCM observatory (Madrid) using AstMon astronomical camera, the SQM and TESS photometers. AstMon is an all sky camera that perform astronomical photometry using standard stars. It provides all sky maps in the Johnson B, V and R photometry bands. Preliminary results of the field test show that TESS-W photometer is around 0.5 magnitudes more sensible than the Unihedron SQM photometer on polluted skies as Madrid: using a zero point $\mathrm{ZP}=20.38$ for TESS the curves of SQM $(\mathrm{ZP}=19.9)$ are similar. 
The graphs of Figure 5 show the dependence of this offset with (B-V) and (V-R) colors (first two panels) and the variation of the color of the sky in Madrid along this night. The color of the sky changes along the night in Madrid as the bluer ornamental lights are being switched off. The offset between SQM and TESS depends on the color of the sky. This an expected result since the TESS spectral response is extended to the red to cover the High Pressure Sodium (HPS) lines (around $590 \mathrm{~nm}$ ) that dominates the Madrid spectrum (Figure 3, panel 6), while the SQM bandwidth decreases at the wavelength of the HPS lines.

We also tested the TESS photometer taking measurements from a moving vehicle. The results are comparable to those obtained with an SQM measuring at the same time. This method is useful to build night sky brightness maps as the one made around Madrid using SQM photometers [8].

\section{Continuing Work}

Up to now, the hardware and communication software of the TESS-W photometer are working without any problem. After the successful test the next 70 units are being assembled. The photometers will be cross calibrated on the LICA-UCM optical lab. We hope that the photometers will be ready for distribution in the next months. We are developing more low cost devices that could measure night sky brightness [13].

Measuring night sky brightness with photometers pointing to the zenith is a first estimate of the light pollution effect at this location. However, it is a simple and reliable method to monitor the evolution of the sky brightness. Analyzing the data taken along the night, every night along extended periods it is possible to obtain statistical results of the variation of the sky brightness with atmospheric conditions [1],[2],[12].

More detailed information about the color and location of the sources can be obtained with all-sky images taken with cameras fitted with fish-eye lenses that are more complicated and time consuming. For a complete description of the methods it is recommended to read the review of A. Hänel [9]. All-sky brightness maps can also be built using simple photometers and the NixNox project method [10][11].

Interested citizens will help us in monitoring the light pollution of European villages using fixed photometers pointing to the zenith ('European Photometer Network'). The aim is to extend the photometer network of the Spanish Light Pollution research network [12] to European countries. We plan to joint efforts with other networks in Europe with similar monitor stations (Italy, Switzerland, etc.). The data collected will be archived in open repositories. The citizens, associations, and schools will be able to purchase and install low cost night sky brightness photometers, which will be sold in the STARS4ALL marketplace.

\section{Acknowledgements}

STARS4ALL is a project funded by the European Union H2020-ICT-2015-688135. This work has been partially funded by the Spanish MICINN (AYA2012-30717, AYA2012-31277 and AY2013-46724-P), by the Spanish program of International Campus of Excellence Moncloa (CEI), and the Madrid Regional Government through the SpaceTec Project S2013/ICE-2822, The support of the Spanish Network for Light Pollution Studies (Ministerio de Economía y Competitividad (MINECO) AYA2015-71542-REDT) is also acknowledged.

\section{References}

[1] Bará, S. (2016). Anthropogenic disruption of the night sky darkness in urban and rural areas. Royal Society Open Science, 3(10), 160541.

[2] Kyba, C. C., Tong, K. P., Bennie, J., Birriel, I., Birriel, J. J., Cool, A., ... \& Ehlert, R. (2015). Worldwide variations in artificial skyglow. Scientific reports, 5, 8409.

[3] Cinzano, P. (2005). Night sky photometry with sky quality meter. ISTIL Int. Rep, 9.

[4] Falchi, F., Cinzano, P., Duriscoe, D., Kyba, C. C., Elvidge, C. D., Baugh, K., ... \& Furgoni, R. (2016). The new world atlas of artificial night sky brightness. Science advances, 2(6), e1600377.

[5] European Research Project - STARS4ALL H2020-ICT-2015. Available at: http://www.stars4all.eu/

[6] Sánchez, S. F., Aceituno, J., Thiele, U., Pérez-Ramírez, D., \& Alves, J. (2007). The night sky at the Calar Alto observatory. Publications of the Astronomical Society of the Pacific, 119(860), 1186.

[7] Aubé, M., Kocifaj, M., Zamorano, J., Lamphar, H. S., \& de Miguel, A. S. (2016). The spectral amplification effect of clouds to the night sky radiance in Madrid. Journal of Quantitative Spectroscopy and Radiative Transfer, 181, 11-23.

[8] Zamorano, J., de Miguel, A. S., Ocaña, F., Pila-Diez, B., Castaño, J. G., Pascual, S., ... \& Nievas, M. (2016). Testing sky brightness models against radial dependency: A dense two dimensional survey around the city of Madrid, Spain. Journal of Quantitative Spectroscopy and Radiative Transfer, 181, 52-66.

[9] Andreas, H. (2015). Quantifying Sky Quality Measurement Methods. ALAN Sherbrooke. 
[10] Zamorano, J., Sánchez de Miguel, A., Alfaro, E., Martınez-Delgado, D., Ocana, F., Nievas, M., \& Gómez Castano, J. (2013). NIXNOX project: Enjoy the dark skies of Spain. Highlights of Spanish Astrophysics VII], Guirado, JC, Lara, LM, Quilis, V., and Gorgas, J., eds, 962-970.

[11] Zamorano Calvo, J., Sánchez de Miguel, A., Nievas Rosillo, M., \& Tapia Ayuga, C. (2014). NixNox procedure to build Night Sky Brightness maps from SQM photometers observations. Available at: http://eprints.ucm.es/26982/ Universidad Complutense de Madrid (Spain).

[12] Zamorano Calvo, J., Sánchez de Miguel, A., Nievas Rosillo, M., Tapia Ayuga, C., Ocaña González, F., Izquierdo Gómez, J., Gallego, J., Pascual, S., Colomer, F., Bará, S., Ribas, S. Morales-Rubio, A., Marco, E., Baixeras, J., Muñoz-Tuñón, C., Díaz Castro, J., Solano, E., Alacid, J.M., Naves, R., Salto, J. L., Luque, S., García, F., Quejigo, R., Ribas, J., Díez, R., García, C., Jáuregui, F. (2015). Light Pollution Spanish REECL SQM Network.

[13] Zamorano, J., Nievas, M., Sánchez de Miguel, A., Tapia, C., García, C., Pascual, S., ... \& Gallego, J. (2015). Low-cost photometers and open source software for light pollution research. IAU General Assembly, 22, 54626 . 